\title{
Study on the Time characteristic of Terahertz for Rubber Material
}

\author{
Feng $\mathrm{Xu}^{1, \mathrm{a}}$, Qingduo Duanmu $\mathrm{u}^{2, \mathrm{~b}}$
}

${ }^{1}$ Changchun University of Science and Technology, College of science, Changchun, Jilin, 130022

${ }^{2}$ Sichuan Vocational and Technical College, Suining, Sichuan, 629000

Keywords: Terahertz; Non-Destructive Testing; Rubber Thickness Uniformity

\begin{abstract}
The Terahertz wave interacts with different media. The detector detects the terahertz time domain waveforms carrying the media information at different time nodes, analyzes the flight time difference of the peak points of different time domain waveforms, and establishes the transmission and reflection Terahertz nondestructive testing Single point thickness extraction model. In the process of flexible assembly, the refractive index of rubber is difficult to be extracted. Based on the single point thickness extraction model, the discrepancy of rubber spatial distribution is proposed by using the standard deviation of thickness uniformity. Terahertz time domain spectroscopy system analysis of rubber thickness uniformity, detection accuracy of up to $100 \mu \mathrm{m}$, to ensure the strength of rubber connection is important.
\end{abstract}

\section{Introduction}

Terahertz time domain spectroscopy (THz-TDS) is a new Terahertz spectral measurement technique developed on the basis of ultrashort pulse technology in recent years. By analyzing the Terahertz pulse time-resolved electric field between the transmitted sample and the equivalent length in the free space the relative change [1]. At present, the THz-TDS system is used to analyze the material, mainly in order to obtain the physical and chemical parameters such as the complex permittivity, dispersion and absorption of the measured material in the Terahertz band, and use these parameters to study the composition, structure and mutual Role relationship [2-4], but has not yet carried out the application of THz-TDS system thickness of the uniformity of the relevant research.

\section{Terahertz Nondestructive Testing Thickness Uniformity Detection}

The interaction between electromagnetic waves and matter is usually described by the complex refractive index of the material, $\mathrm{n}=\mathrm{n}+\mathrm{i} \beta$, which is a function of wavelength. The real part $\mathrm{n}$ and the imaginary part $\beta$ of the complex refractive index are the refractive index and the extinction coefficient, respectively. In a homogeneous isotropic medium, propagating in the z-direction, the electromagnetic wave with the wavelength $\lambda$ can be expressed as:

$$
E(x)=E_{0} \exp \left(-\frac{2 \pi \beta z}{\lambda}\right) \exp \left[\frac{2 \pi}{\lambda} i(i z-c t)\right]
$$

The Terahertz wave also belongs to the electromagnetic wave that propagates in the same way in the medium. Due to the selective penetration of the Terahertz wave to the material, different substances have different responses in the Terahertz band, and many nonpolar dielectric materials are transparent to the Terahertz radiation, but the Terahertz wave is reflected by the metallic material [6]. Therefore, according to the nature of the experimental material, select the transmission and reflection of the experimental way to detect the uniformity of sample thickness.

The Terahertz wave is transmitted at an angle $\theta \mathrm{i}$, and the Terahertz wave is generated at the interface of the air-medium interface, and the Terahertz wave is generated by the Terahertz wave at the interface of the air- The reference signal; the transmitted Terahertz wave, reflected on the lower surface of the sample, detects the Terahertz echo carrying the sample information.

According to the theory of Terahertz wave propagation, a reflection type single point thickness extraction model is established 


$$
\begin{aligned}
d & =\frac{c \sqrt{n^{2}-n_{0}^{2} \sin ^{2} \theta_{i}}}{2 n}\left(T_{\text {dears }}-T_{\text {app }}\right) \\
& =\frac{c \sqrt{n^{2}-n_{0}^{2} \sin ^{2} \theta_{i}}}{2 n} \Delta T
\end{aligned}
$$

When the Terahertz wave is incident vertically, the single-point thickness extraction model can be simplified as:

$$
d={ }_{2 n}^{c}\left(T_{\text {deas }}-T_{\text {sp }}\right)={ }_{2 n}^{c} \Delta T
$$

Where $\mathrm{d}$ is the thickness of the sample; Tup and Tdown are Eup, Edown's flight time, $\mathrm{n}$ is the refractive index of the sample, and c is the propagation velocity of light in the air.

Similarly, the transmission type single point thickness extraction model is established by detecting the reference signal Eref and the transmitted signal Esam of the transmitted air and the measured sample. Equation (4) shows that the flight time difference is a direct representation of the optical path change:

$$
\left.d=\frac{c}{n-n_{0}} T_{\text {sem }}-T_{r f}\right)=\frac{c}{n-n_{0}} \Delta T
$$

Among them, the air and sample refractive index were $\mathrm{n} 0$ and $\mathrm{n}$; Tsam and Tref were Eref, Esam's flight time.

In the case where the refractive index of the sample is known, the sample thickness can be accurately obtained by detecting the flight time difference of the Terahertz echo. However, generally in the engineering test, the optical parameters of the sample is difficult to extract, and the echo time difference can reflect the thickness of the measured sample information, therefore, with the reflection of the thickness of the correlation coefficient $\rho$ simplified thickness model:

$$
d=\rho \cdot \Delta T
$$

\section{Experimental and Data Analysis}

Experimental Device. Experiment with the United States Picometrix T-Ray5000 system. The system is a compact, self-contained time domain system with highly integrated, light-emitting antenna method for generating and detecting Terahertz pulses, consisting mainly of femtosecond lasers, Terahertz emitter components, Terahertz detection elements, and time delay systems. The THz-TDS system pulse width of $0.1 \sim 3.5 \mathrm{THz}$, signal to noise ratio $>70 \mathrm{~dB}$ (frequency domain $1 \mathrm{~h}$ average), fast scanning range of 320ps, scanning frequency of $100 \mathrm{~Hz}$, spectral resolution $<8 \mathrm{GHz}$, depth resolution of $100 \mu \mathrm{m}$.

Thickness Uniformity Analysis. Rubber adhesives are commonly used in the field of composite material production and flexible assembly. As the connecting material of composite material and metal component flexible assembly, the thickness of rubber not only determines the connection strength, but also affects the service life. Therefore, it is very important to analyze the uniformity of rubber thickness.

In order to avoid the influence of the composite material on the experimental results, the rubber adhesive was directly applied to the polished aluminum plate (the aluminum plate was completely covered), the aluminum plate size was $50 \mathrm{~mm} \times 50 \mathrm{~mm}$, the rubber thickness was about For $2 \mathrm{~mm}$. In order to make the experimental results can accurately reflect the changes in rubber thickness, in the coating process and the rubber center area was artificially thickened.

The experiment was carried out under the condition of room temperature $\left(25^{\circ} \mathrm{C}\right)$ and air humidity of $20 \%$. Since the Terahertz pulse could not transmit the aluminum plate, the experiment was carried out by using the reflection detection method, and the measured space was detected by point-by-point scanning. Because of the difficulty in making the standard parts of rubber distribution, it is difficult to extract the reflection thickness correlation coefficient. Therefore, the thickness extraction model is simplified by the method of setting the thickness correlation coefficient. Which can clearly distinguish the rubber thickness uniformity of information, and the change is very obvious, indicating that the central area of rubber thicker, the edge of rubber thin. The degree of change in thickness relative to the mean can be quantitatively analyzed from 
chromaticity changes.

\section{Conclusion}

In this paper, the single point thickness extraction model is simplified by the method of detecting the standard parts in the case where the refractive index of the composite standard parts is unknown, and the thickness model is universal. The flexible assembly process of composite material and metal member was simulated in the laboratory. The uniformity detection of rubber was carried out by point-by-point scanning method. The spatial distribution of rubber was obtained, and the three-dimensional topography of rubber spatial distribution was drawn. The uniformity of rubber thickness is reflected, and the dispersion of rubber spatial distribution is proposed by using the standard deviation of thickness uniformity. THz-TDS system is used to analyze the thickness uniformity. It has the advantages of high precision, simple operation and no harm to the human body. It can be used for real-time detection of composite material production and flexible assembly process, which is beneficial to reduce the production cycle and ensure the quality of the project.

\section{References}

[1] Li Jingzhen. Hand book of optics [M]. Xi'an: Xi'an science and technology press, 2010: 371-401

[2] Zhang Cunlin, Mukaijun. Terahertz spectroscopy and imaging [J]. Laser and Optoelectronics Progress, 2010, 02: 1-14

[3] WangXiumin. Determine sample thickness in the process of terahertz spectrum analysis using error theory [J]. Laser and Infrared, 2009, 39(8): 884-886

[4] Chen Liqun, Gao Fei, Gong Xiaojing. Extraction of refractive indices of materials through transmission terahertz time-domain spectroscopy [J]. Journal of Infrared and Millimeter Waves, 2013, 02: 160-164

[5] Lionel Duvillaret, Frederic Garet, Jean-Louis Coutaz. Highly precise determination of optical constants and sample thickness in terahertz time-domain spectroscop [J]. Applied Optics, 1999, 1

[6] Ryan Michae Smith. Terahertz frequency analysis of gaseous and solid samples using Terahertz time - domain spectroscopy [D]. University of Iowa, 2012 